\title{
Study on Dynamic Characteristics and Parameter Influence of a Kind of Bilateral Restraint Impact Vibration System
}

\author{
Li Ding $\left(\mathbb{D},{ }^{1}\right.$ Shangshang Cheng $\mathbb{D},{ }^{1}$ Kaifei Zhang, ${ }^{1}$ Jiajun Chai, ${ }^{1}$ He Li $\mathbb{D},{ }^{1}$ Chengdong Niu, ${ }^{2}$ \\ and Chao $\mathrm{Cao}^{2}$
}

${ }^{1}$ College of Mechanical and Electrical Engineering, Henan Agricultural University, Zhengzhou 450002, China

${ }^{2}$ College of Engineering, China Agricultural University, Beijing 100083, China

Correspondence should be addressed to He Li; chungbuk@163.com

Received 31 March 2021; Revised 26 October 2021; Accepted 25 November 2021; Published 16 December 2021

Academic Editor: Fabio Rizzo

Copyright (C $2021 \mathrm{Li}$ Ding et al. This is an open access article distributed under the Creative Commons Attribution License, which permits unrestricted use, distribution, and reproduction in any medium, provided the original work is properly cited.

\begin{abstract}
In this paper, the hinge in the articulated structure is studied, the gap hinge is described as a nonlinear bilateral constraint, and the equivalent modeling and analysis of the hinge connection collision vibration are carried out based on the Lankarani-Nikravesh nonlinear contact force model. With the help of the method of nonlinear system dynamics analysis research, the Poincaré mapping of hinge joint collision vibration is constructed, the bifurcation diagram of the system with different parameters is solved, and the variation law of the system motion and the influence of parameters are analyzed by combining the time response diagram, phase diagram, Poincaré cross section diagram, and spectrum diagram of the typical motion of the system. The simulation results show that the system moves in a single degree of freedom and varies with parameters with multiplicative period bifurcation and rubbing edge bifurcation leading to chaos; the system's periodic motion has shock state mutation and mirror jump transformation.
\end{abstract}

\section{Introduction}

The hinge connection is a basic form of mechanical connection commonly found in folding trusses, folding wings, and large antennas. The hinged structure transmits loads mainly through compression and friction between contact interfaces. Gaps or restraints in the hinged structure are ubiquitous due to the need for motion limitation, thermal expansion, and cold contraction of certain parts of the mechanical components [1-4].

Local gap collisions are prone to large shock dynamic loads and high-frequency vibrations, which increase noise levels and exacerbate wear and tear of mechanical components, posing a huge risk to the reliability and stability of the connected structure. For example, in the 1990s, the Hubble telescope in the United States suffered from periodic "jamslip" failures caused by thermal vibrations of the support beam and instantaneous vibrations of the gap hinges, resulting in reduced attitude stability and compromised observations. The study of vibration-impact dynamics is relevant to the design of dynamic performance and noise suppression of impact mechanisms and mechanical systems with motion-limiting constraints, and a large number of studies on the dynamics of connection structures have been conducted since the 1970s. Structural dynamics experiments have shown that the stiffness and damping of hinge-connected structures have nonlinear properties such as discontinuity, hysteresis, and segmental linearity [5-12]. Gritli et al. found in a feedback system based on the OGY control method that in a two-parameter bifurcation diagram, the boundary collision bifurcation occurs within a bounded interval of nominal parameters [10].

Andreaus et al. established the equations of motion with and without dimensionality when acceleration and two unidirectional constraints were applied to the experimental object and eventually found that only a unilateral constraint was needed to change the dynamics of the system. Stefani et al. introduced suitable dimensionless parameters in their experiments. De Angelis et al. experimentally investigated the effect of geometric and mechanical properties of 
vibration isolation and damping devices on the nonlinear, nonsmooth response of vibration shock systems [13-22]. De Angelis et al. investigated the dynamic response of an SDOF oscillator under a fundamental acceleration excitation.

Guo et al. showed that the adhesion time would be negatively correlated with the shock intensity for a periodic two-degree-of-freedom shock. Wang et al. found that decreasing the intrinsic frequency would consume less shock energy and make the vibration frequency smaller. Based on the theory of discontinuous dynamics, Chen et al. described in detail the chaotic motion and periodic trajectory under different parameters and initial conditions through numerical simulations. $\mathrm{Li}$ et al. discussed the global dynamics of a nonsmooth dynamics model under the joint action of three nonsmooth factors, namely elastic shock, rigid shock, and dry friction [23-31]. Fan et al. conducted a discontinuous dynamic analysis of a class of three-degree-of-freedom mechanical oscillation systems with dry friction and unilateral rigid shocks, revealing the complex switching mechanism of object motion in discontinuous dynamical systems.

The focus of this paper will be on the description of the mechanical behavior of the hinge gap and contact interface. In Section 2, the end hinge collision vibration equivalent model is established by combining the hinge radial contact collision case, the Poincaré mapping of the system containing the gap is constructed, and the system bifurcation diagram and Lyapunov exponent under each control parameter, respectively, are solved. In Section 3, dimensionless quantities are introduced into the motion of the established end-hinge collision system through differential equations and nonlinear contact force expressions into the established end-hinge collision system, which constructs a system of differential equations and numerically simulates the dynamics of end-hinge gap collision. In Section 4, the effects of the connection structure parameters on the nonlinear characteristics of the system are compared and analyzed, which will be investigated to provide practical guidance for optimizing the design of the connection structure.

\section{Materials and Methods}

2.1. Equivalent Modeling of Hinge Connection Impact Vibration. As shown in Figure 1, the hinge is composed of a double ear bar, a single ear bar, a pin shaft, and a shaft sleeve, and the elastic thin wall is used to simulate the bar connection constraints. The pin shaft is fixed with the double ear bar, and the shaft sleeve is fixed with the single ear bar [32]. Considering the radial contact collision problem of the hinge caused by the clearance between the pin shaft and the shaft sleeve, the equivalent collision vibration model of the hinge is established, as shown in Figure 2.

In the model, the elastic thin wall is simplified into linear spring and linear damping, the double ear bar and single ear bar are defined as concentrated masses, and the clearance contact of the hinge is treated as a nonlinear bilateral constraint.

To analyze the vibration characteristics of the gap impact of the hinge connection, a simple harmonic excitation force is applied to the oscillator in Figure 2. When the oscillator moves to the nonlinear constraint boundary, a contact

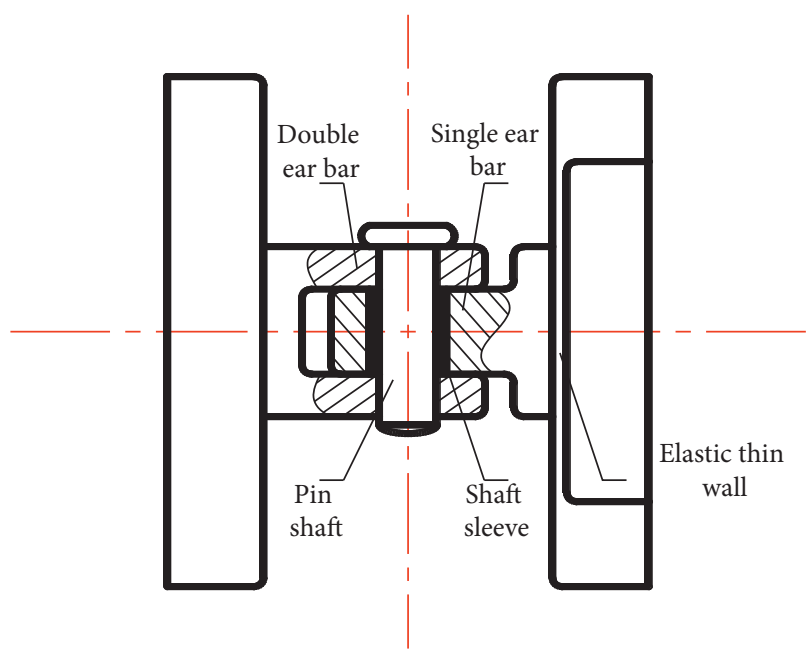

FIgURE 1: Hinge connection form.

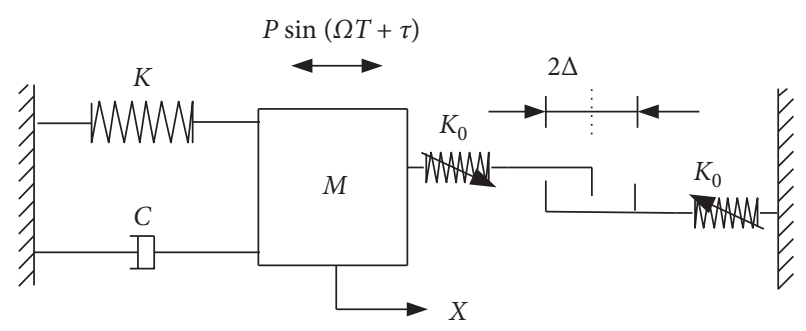

Figure 2: Equivalent dynamic model of the hinge connection.

collision will occur. After a certain period, it will move in the opposite direction, and so on. The differential formula of motion can be expressed as

$$
M \ddot{X}+C \dot{X}+K X+F_{N L}(X, \dot{X})=P \sin (\Omega T+\Gamma) .
$$

In formula (1), $F_{N L}(X, \dot{X})$ is the interaction force at the gap hinge in the vibration process, which has nonlinear characteristics.

2.2. Nonlinear Contact Force, Poincaré Mapping, and Lyapunov Exponent. For the system with clearance, the gap constraint makes the trajectory in the phase space of the system different from the smooth nonlinear system: the trajectory crosses the constrained boundary, the dynamic formula of the system is switched, the smoothness of the trajectory is destroyed, and the contact may cause singularity Two different methods for constructing Poincaré mapping of systems with gaps involves two different methods of mapping cross sections in phase space: the fixed phase cross section and the constrained boundary determined by the Poincaré cross section [33]. Two kinds of Poincaré mapping of the system are constructed based on two kinds of Poincaré mapping cross sections.

2.2.1. Nonlinear Contact Force of Clearance Hinge System. The Lankarani-Nikravesh contact force model (L-N model), which is widely used in current research, is selected for the impact contact force of the clearance hinge: 


$$
F=K \delta^{n}+c \delta^{n} \dot{\delta}=K \delta^{n}\left[1+\frac{3\left(1-c_{e}^{2}\right)}{4} \frac{\dot{\delta}}{\dot{\delta}^{(-)}}\right] .
$$

In formula (2), the symbol $\delta$ means deformation amount, $\dot{\delta}$ means deformation velocity, $n$ is the nonlinear index, $\mathrm{K}$ is contact stiffness coefficient, $\dot{\delta}^{(-)}$means initial velocity, $c_{e}$ is recovery coefficient, and $n$ is the nonlinear index. Take the nonlinear exponent $n=1.5 . \mathrm{K}$ is the equivalent contact stiffness, which is as follows:

$$
\begin{aligned}
K & =\frac{4}{3 \pi\left(\sigma_{B}+\sigma_{J}\right)} \sqrt{\frac{R_{B} R_{J}}{R_{B}-R_{J}}} \\
& =\frac{4}{3} E^{*} \sqrt{R^{*}},
\end{aligned}
$$

where $c$ is the viscous damping coefficient, which is determined by the formula of energy dissipation and damping work in the collision process, which is as follows:

$$
\Delta E=\frac{1}{2} m_{e}\left[\dot{\delta}^{(-)^{2}}-\left(c_{e} \dot{\delta}^{(-)^{2}}\right)\right]=\oint c \delta^{n} \dot{\delta} \mathrm{d} \delta .
$$

In formula (4), $m_{e}$ is the equivalent mass : $m_{e}=m_{B} m_{J} /\left(m_{B}+m_{J}\right)$. And the viscous damping factor can be obtained from the following equation:

$$
c=\frac{3 K\left(1-c_{e}^{2}\right)}{4 \dot{\delta}^{(-)}} .
$$

The L-N model clearly defines the energy loss and transfer in the process of contact collision and takes into account the influence of contact surface shape, material, and collision velocity. It is widely used by foreign and domestic researchers in the study of collision dynamics of systems with clearance. Therefore, the dynamic analysis of hinge joint clearance collision is also based on this model.

\subsubsection{Construction of Poincaré Maps for Systems with Gaps.} For the system with clearance, the trajectory in the phase space of the system is different from that of the smooth nonlinear system because of the clearance constraint: the trajectory passes through the constrained boundary, the dynamic formulas of the system switch, the smoothness of the trajectory is destroyed, and the contact may cause singularity. The construction of Poincaré mapping for systems with gaps involves two different methods of mapping cross sections in phase space [34] : the fixed phase section and the constraint boundary determined by the Poincaré sections. Two kinds of Poincaré maps are constructed based on two kinds of Poincaré cross sections: (1) displacement map based on fixed phase cross section and (2) shock map based on switching surface. The fixed phase displacement mapping can directly determine the number of collision periods, and the impact mapping can determine the impact number of the gap hinge and the corresponding constraint boundary.

The model is simplified by bilateral nonlinear constraints with clearance hinge connection, and the periodic motion of the system is represented by the symbol "n-p-q," where $n$ is the number of excitation periods contained in the motion of the system, $\underline{P}$ and $Q$ are the numbers of collisions with the left and right boundaries of the nonlinear constraint.

Poincaré's mapping is based on a system with gaps. The left and right boundary constraint surfaces and any fixed phase surfaces are selected as Poincaré sections $\sigma_{p}, \sigma_{q}$, and $\sigma_{n}$, respectively. Poincaré mappings are established for bilaterally restricted oscillation systems. For periodic and subharmonic impact vibrations, the fixed points $p$ and $q$ on Poincare sections $\sigma_{p}$ and $\sigma_{q}$ represent the impact times of the oscillator, the left boundary, and the right boundary in the movement period of the system. The fixed point $n$ on Poincaré section $\sigma_{n}$ is equal to the ratio of the period of motion of the system to the period of excitation force. For quasiperiodic and chaotic motions, Poincaré sections $\sigma_{p}, \sigma_{q}$, and $\sigma_{n}$ are dense set of points, which can effectively characterize the attractor of the system.

\subsubsection{Wolf Calculation Method of Lyapunov Exponent.} In the study of the bifurcation and chaos of nonlinear systems with clearance impact vibration, the positive and negative sum of the largest Lyapunov exponent is often used as a reliable criterion for the existence and strength of chaos. The Wolf method $[35,36]$ is used to calculate the maximum Lyapunov exponent of the impact vibration system with gap hinge connection, and the Lyapunov exponent is estimated through the evolution process of phase trajectory, phase plane, and phase volume. The following details the specific steps of Wolf method to calculate the maximum Lyapunov exponent:

(1) Phase Space Reconstruction. For a chaotic time series $X(t)=\left\{x_{1}, x_{2}, \ldots, x_{N}\right\}$, the phase space is reconstructed from the embedding dimension $m$ and the delay time $\tau$ :

$$
\begin{aligned}
X(t) & =\left\{x_{1}, x_{2}, \ldots, x_{N}\right\}, \\
Y(t) & =\left\{X_{1}(t), X_{2}(t), \ldots, X_{i}(t), \ldots, X_{m}(t)\right\} .
\end{aligned}
$$

$$
\text { In formula }(6), \quad X_{i}(t)=\left\{x_{1+\tau(i-1)}, x_{2+\tau(i-1)},\right.
$$

(2) Phase Trajectory Evolution. Search for the nearest twophase points at the initial moment in the phase space and record the distance; track the time evolution of the distance to the desired point, at a certain time $\mathrm{tl}$, the distance exceeds the specified value, calculate the exponential growth of the distance; search for the nearest two-phase points at $\mathrm{t} 1$, and make the distance and the included angle as small as possible, as shown in Figure 3; continue the abovementioned process until the end of the time series.

(3) The Calculation of Lyapunov Exponent. Assuming that the number of iterations in step (2) is $M$, the maximum Lyapunov exponent is as follows:

$$
\lambda=\frac{1}{t_{M}-t_{0}} \sum_{i=0}^{M} \ln \frac{L_{i}^{\prime}}{L_{i}} .
$$




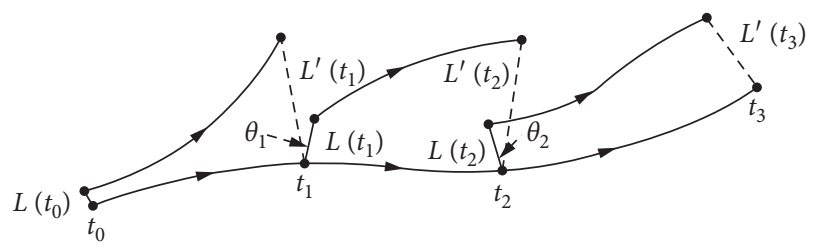

Figure 3: Schematic diagram of the Wolf method for calculating the maximum Lyapunov exponent.

\section{Numerical Simulation of End Hinge Clearance Impact Dynamics}

The differential formula of motion of the end hinge clearance impact system is expressed by formula (1), and the expression of nonlinear contact force is expressed by formula (2).

$$
\begin{aligned}
t & =T \sqrt{\frac{K}{M}}, \\
x & =\frac{K}{P} X, \\
\zeta & =\frac{C}{2 \sqrt{K M}}, \\
\omega & =\Omega \sqrt{\frac{M}{K}}, \\
\tau & =\Gamma, \mu=\frac{3\left(1-c_{e}^{2}\right)}{4}, \\
k_{0} & =\frac{P^{n-1}}{K^{n}} K_{0}, \\
\delta & =\frac{K}{P} \Delta .
\end{aligned}
$$

The differential formulas of the motion of the system are obtained, which are as follows:

$$
\begin{array}{ll}
\ddot{x}+2 \zeta \dot{x}+x+f(x, \dot{x})=\sin (\omega t+\tau), & \\
f= \begin{cases}k_{0}(x-\delta)^{n}\left[1+\mu\left(\frac{\dot{x}}{\dot{x}^{(0)}}\right)\right], & x \geq \delta, \\
0, & -\delta<x<\delta, \\
k_{0}(-x-\delta)^{n}\left[-1+\mu\left(\frac{\dot{x}}{\dot{x}^{(0)}}\right)\right], & x \leq-\delta .\end{cases}
\end{array}
$$

By reducing the order of formula (9), the first-order differential formulas are obtained:

$$
\left\{\begin{array}{l}
\dot{x}=y, \\
\dot{y}=\sin (\omega t+\tau)-2 \zeta y-x-f(x, y) .
\end{array}\right.
$$

Direct numerical integration is carried out with the aid of an ODE differential formula solver in MATLAB. When dealing with the differential formula of the impact vibration system, the state of the system motion to the constraint boundary must be calculated accurately by the idea of the seam method, which is used as the initial value of the next step formula solving to realize the transformation of the relationship between the free motion and the contact collision state [37] In this paper, based on the event-driven method and the variable step fourth-order Runge-Kutta integration algorithm, the solution is programmed in MATLAB.

3.1. Periodic Motion and Bifurcation of Hinge Clearance Impact. For the differential formula of motion of the system shown in formula (10), determine the reference parameter: $k_{0}=300, \zeta=0.2$, and $\delta=0.01$ and take the excitation frequency $f$ as the control parameter to solve the system response. The global bifurcation diagram and the maximum Lyapunov exponent diagram of the system are shown in Figure 4 . In the bifurcation diagram, the abscissa is the excitation frequency and the ordinate is the Poincare map of the velocity at the nonlinear constraint boundary of the system.

3.2. Period-Doubling Bifurcation and the Way to Chaos. From the bifurcation diagram in Figure 4, when the excitation frequency $f=0.41 \sim 0.43 \mathrm{~Hz}$, the system response experienced a typical process from single period motion to period-doubling bifurcation to chaotic motion.

It can be seen from Figure 5 that when $f=0.429 \mathrm{~Hz}$, the displacement and velocity curves in the time history diagram show regular periodic changes, there is a single point in the Poincaré section, the power spectral density diagram is a discrete spectrum, and there is only frequency doubling frequency. Combined with the phase diagram, the system makes a stable bilateral impact asymmetric periodic 1-1-1 motion. As the excitation frequency decreases, the doubling bifurcation occurs when the value of $F$ crosses 0.4265 and 0.4150 , respectively. It is observed from Figures 6 and 7 that when $f=0.422 \mathrm{~Hz}$, there are two fixed points in the Poincaré section, and combined with the phase diagram, the system state evolves into asymmetric periodic 2-2-2 motion; when $f=0.414 \mathrm{~Hz}$, there are four points in the Poincaré section, and the system state evolves into asymmetric periodic 4-4-4 motion, and its power spectral density diagram is similar to that of the same single periodic motion, and the frequency of the whole period-doubling bifurcation process is proportional. The frequency division is mainly concentrated in the main frequency and triple frequency. The excitation frequency continues to decrease, and the system state presents complex motion after the period-doubling bifurcation. It can be seen from the time response diagram and phase diagram in Figures 8 and 9 that when $f=0.410 \mathrm{~Hz}$ and $F=0.405 \mathrm{~Hz}$, the system becomes aperiodic motion, the Poincaré cross section presents dense discrete points, the frequency components of the power spectral density diagram become more and more, fractional frequency gradually appears, and a continuous spectrum appears in the lowfrequency band, combined with the Lyapunov exponent. The results show that the system is in quasiperiodic motion and chaotic motion, respectively, and the system state leads to chaotic motion through period-doubling bifurcation. 


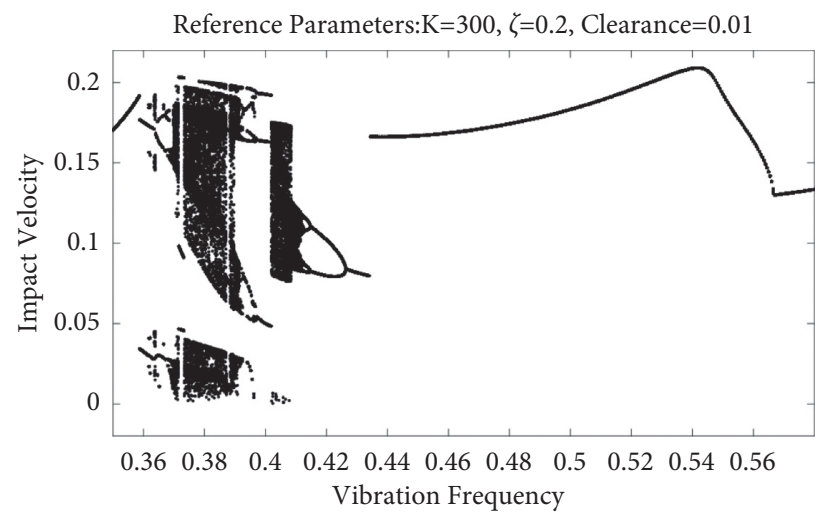

Figure 4: System bifurcation diagram.

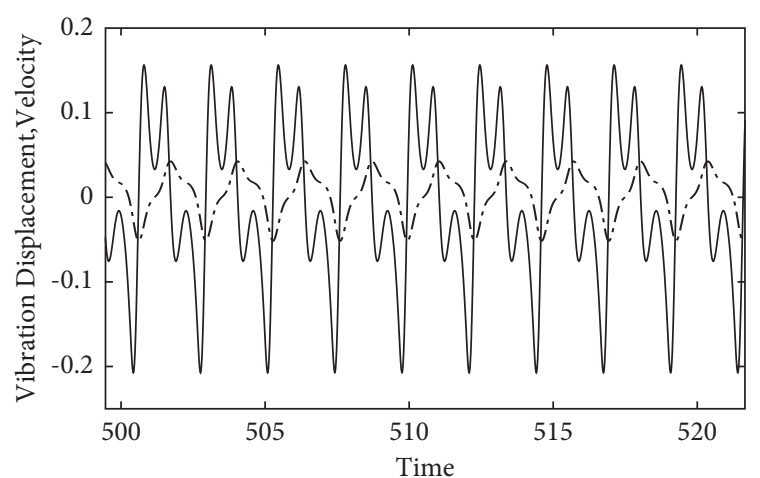

(a)

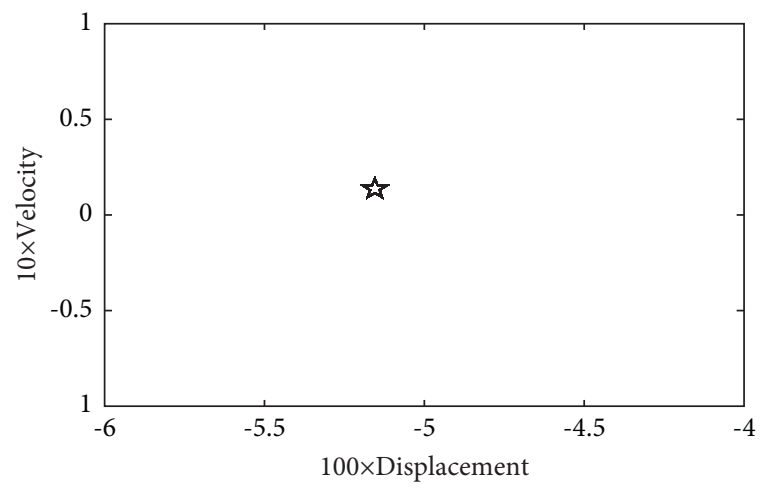

(c)

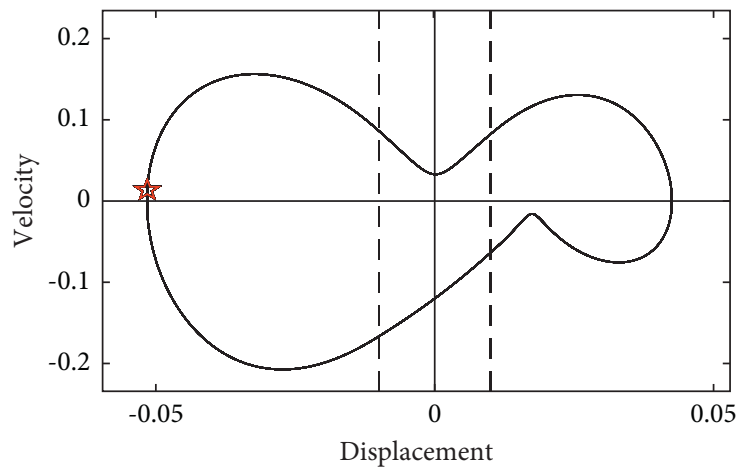

(b)

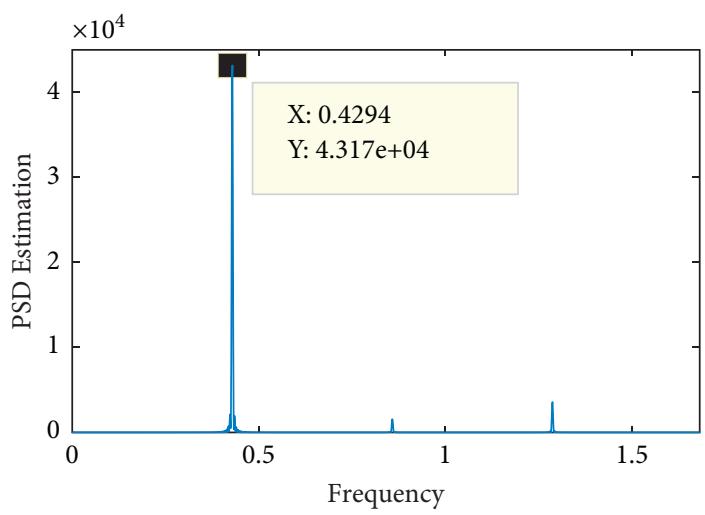

(d)

Figure 5: $f=0.429 \mathrm{~Hz}$, asymmetric period 1-1-1. (a) Time history graph. (b) Phase diagram. (c) Poincaré section. (d) Power spectral density diagram.

3.3. Edge Bifurcations and the Road to Chaos. In the frequency range of $F=0.35-0.37 \mathrm{~Hz}$ and $F=0.389-0.401 \mathrm{~Hz}$, the system appears edge bifurcations and goes into chaos with jump transformation. Taking $f=0.35-0.37 \mathrm{~Hz}$ frequency band as an example, with the increase of excitation frequency, the edging first causes the mirror jumping transformation of the impact state of the periodic motion of the system, and then the periodic motion leads to chaos from the edging bifurcation [38] As shown in Figures 10 and 11, when $f=0.365$ and $f=0.366$, the Poincaré cross section of the system is a single fixed point, which indicates that the motion period of the system is a single period. Compared with the different phase diagrams, the system presents 1-1-2 asymmetric motion and 1-2-1 asymmetric motion, respectively, trimming makes the state of the system undergo mirror jumping transformation. When the frequency continues to increase, from the Poincaré section and phase diagram of $F=0.3674$ and $F=0.368$ in Figures 12 and 13, the 


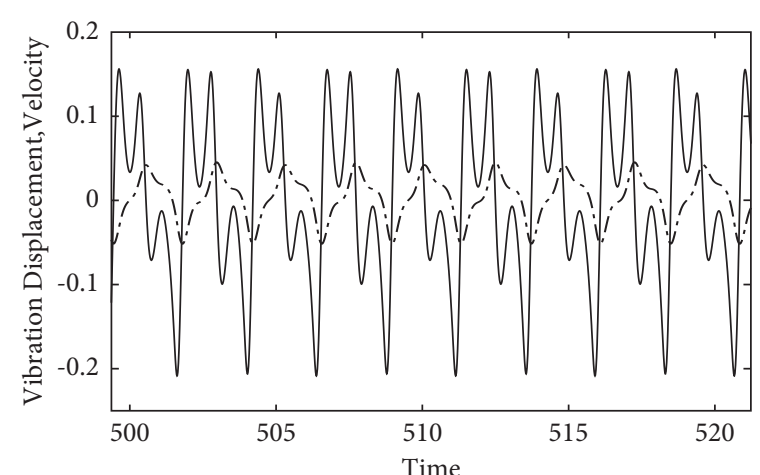

(a)

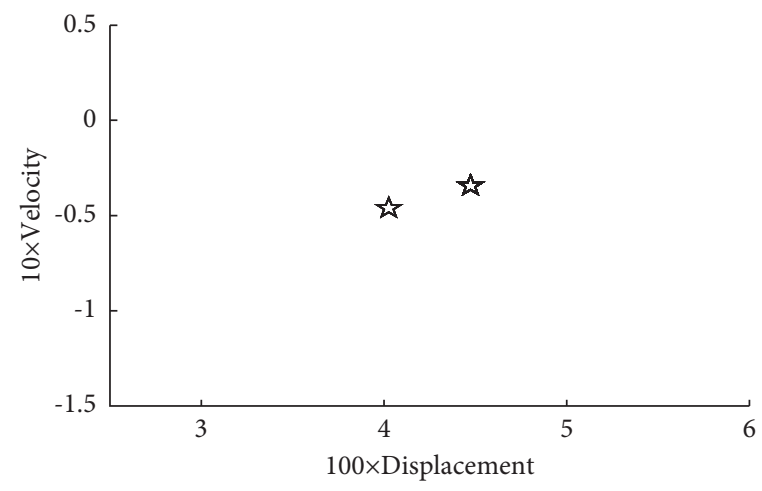

(c)

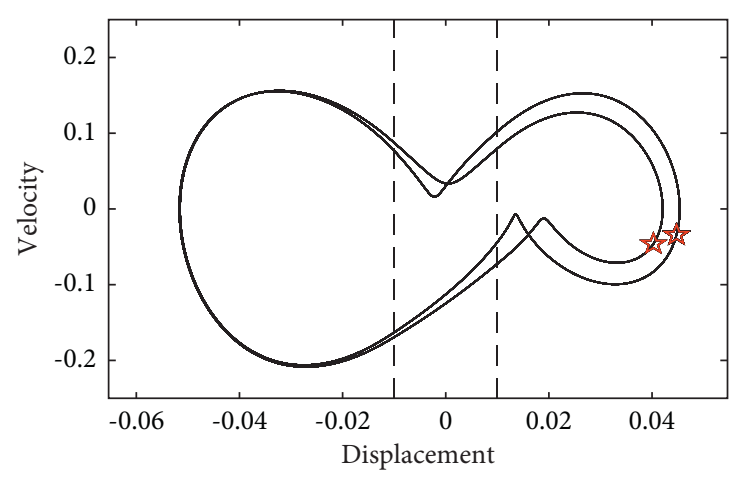

(b)

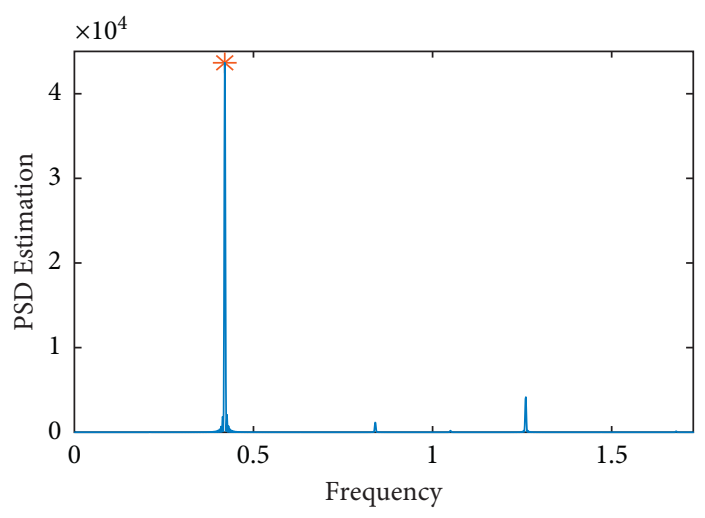

(d)

Figure 6: $f=0.420 \mathrm{~Hz}$, asymmetric period 2-2-2. (a) Time history graph. (b) Phase diagram. (c) Poincaré section. (d) Power spectral density diagram.

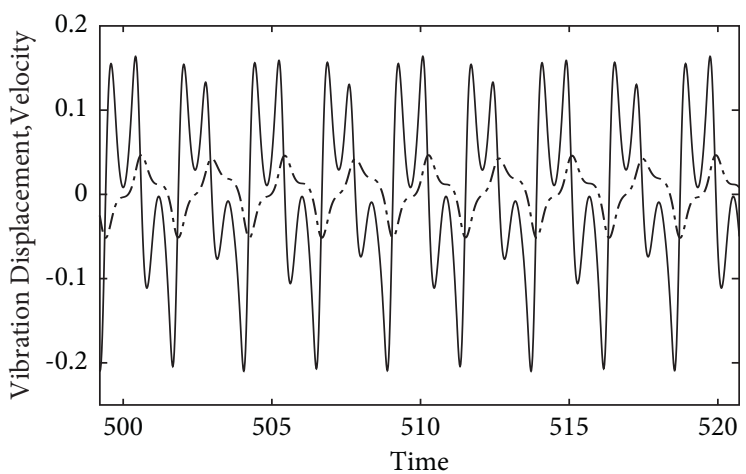

(a)

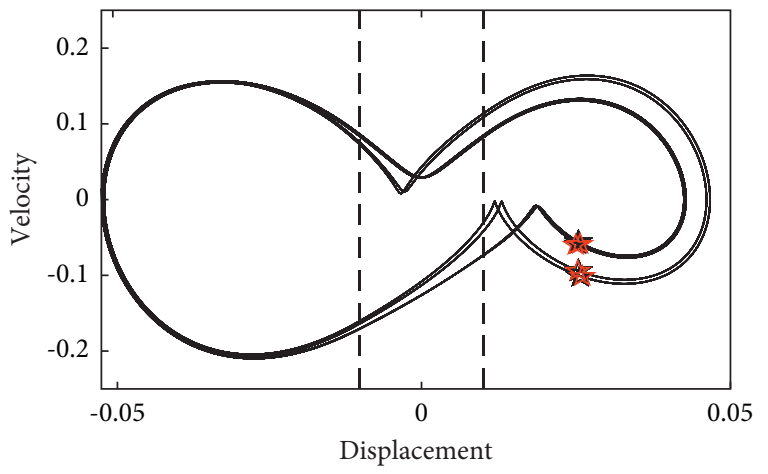

(b)

Figure 7: Continued. 


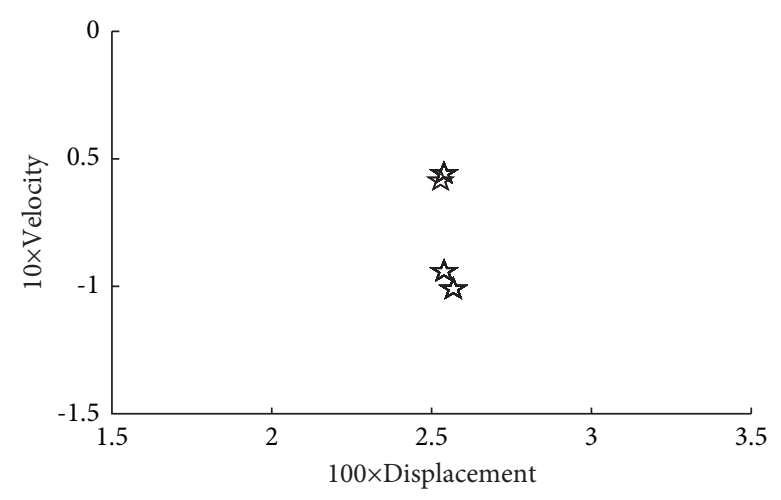

(c)

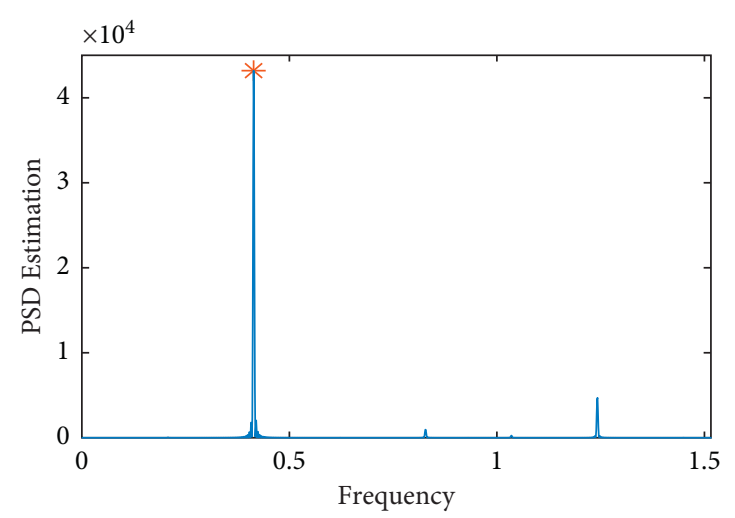

(d)

Figure 7: $f=0.414 \mathrm{~Hz}$, asymmetric period 4-4-4. (a) Time history graph. (b) Phase diagram. (c) Poincaré section. (d) Power spectral density diagram.

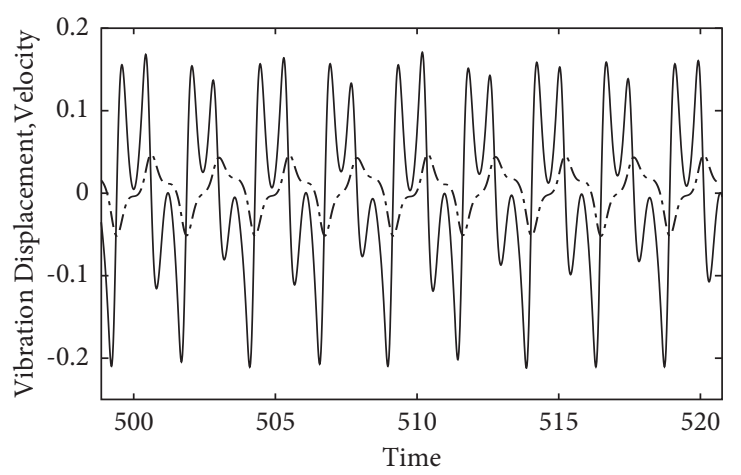

(a)

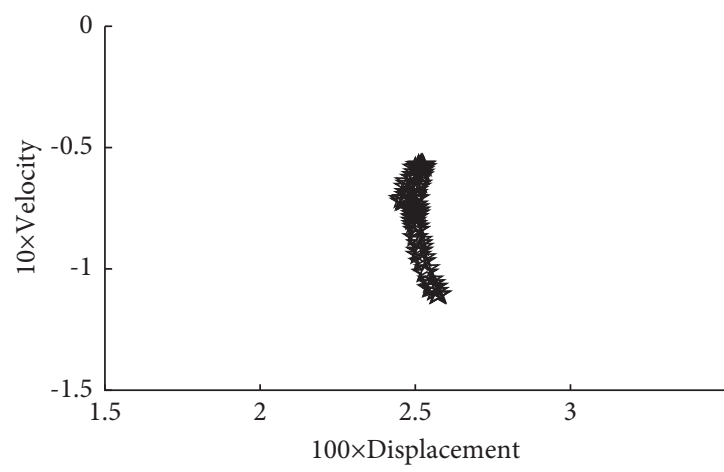

(c)

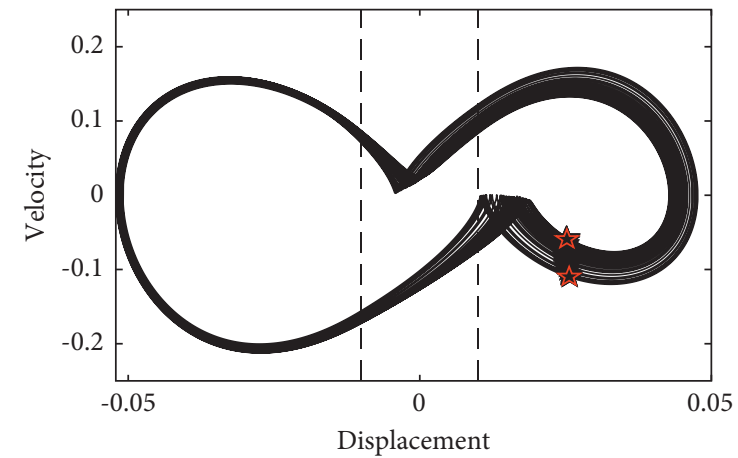

(b)

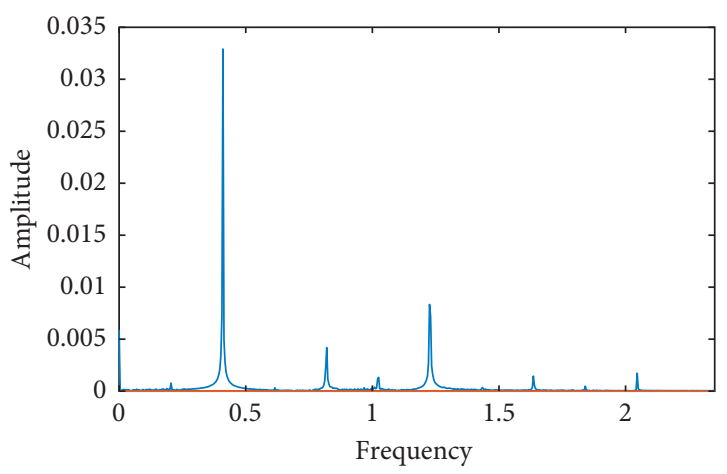

(d)

Figure 8: : $f=0.410 \mathrm{~Hz}$, quasiperiodic motion. (a) Time history graph. (b) Phase diagram. (c) Poincaré section. (d) Power spectral density diagram.

period of the system is doubled, showing 2-4-2 asymmetric motion and 2-2-4 asymmetric motion, respectively. The system undergoes the transition of edge branching and mirror jumping. In Figure 14, when $f=0.3688$, the motion of the system evolves into 4-4-8 asymmetric periodic motion. In Figure 15, when $f=0.370$, the Poincaré cross section is a dense point, and the frequency response is a continuous spectrum. The motion of the system leads to chaos along with the trimming bifurcation and jump transformation.

\section{Influence of Connecting Structure Parameters on Nonlinear Characteristics of the System}

Based on the normalized dynamic differential formula, the structural parameters and system parameters are selected $k_{0}=300, \delta=0.01, \zeta=0.2, c_{e}=0.9$, and $f=0.402,0.414$, $0.426 \mathrm{~Hz}$ as the benchmark parameters, the corresponding dynamic characteristics are taken as the reference results, 


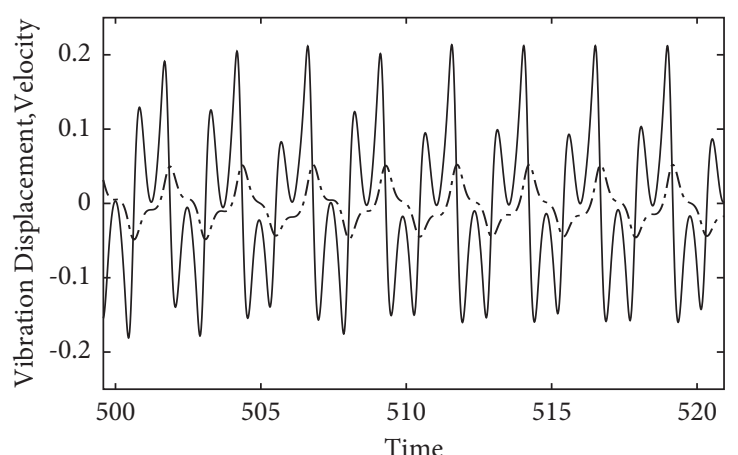

(a)

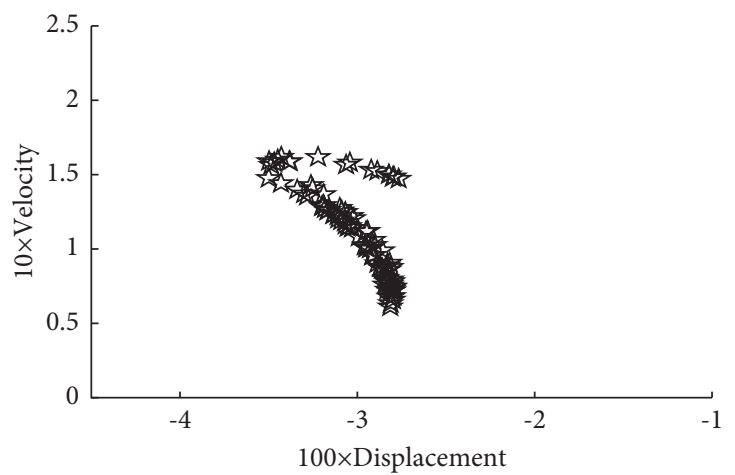

(c)

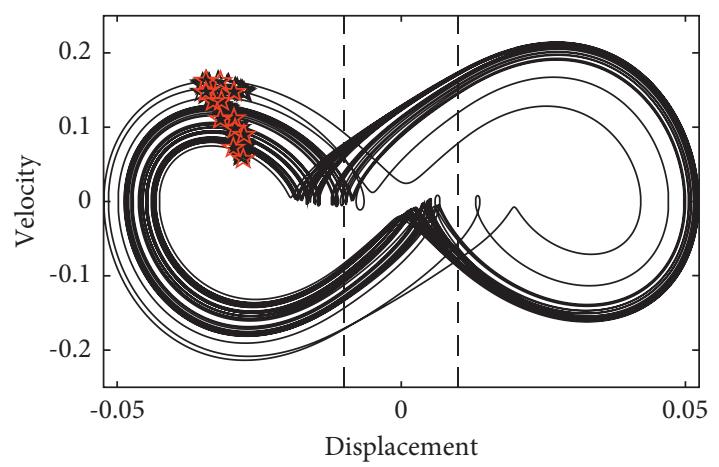

(b)

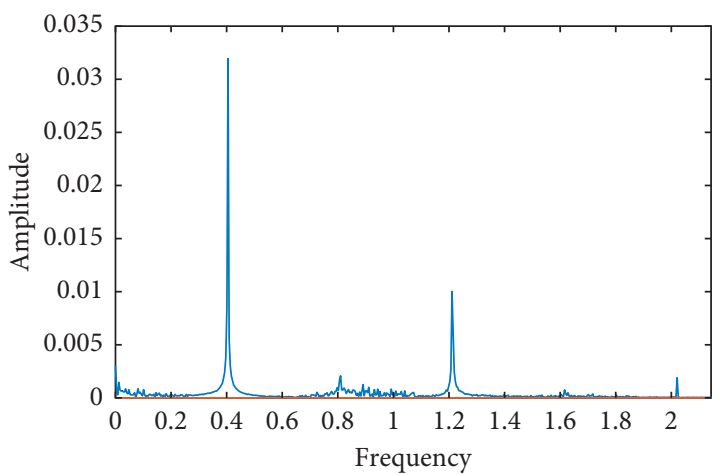

(d)

Figure 9: $f=0.405 \mathrm{~Hz}$, chaotic motion. (a) Time history graph. (b) Phase diagram. (c) Poincaré section. (d) Power spectral density diagram.

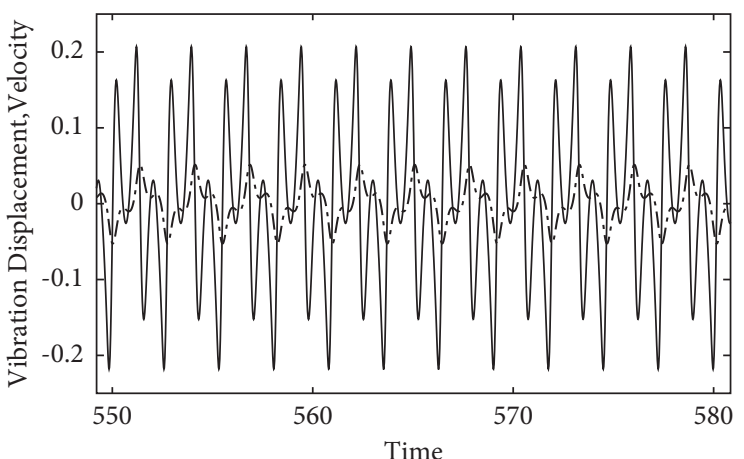

(a)

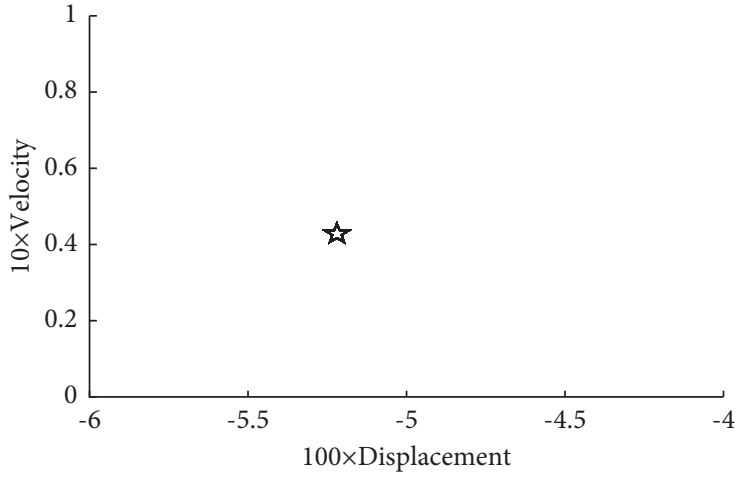

(c)

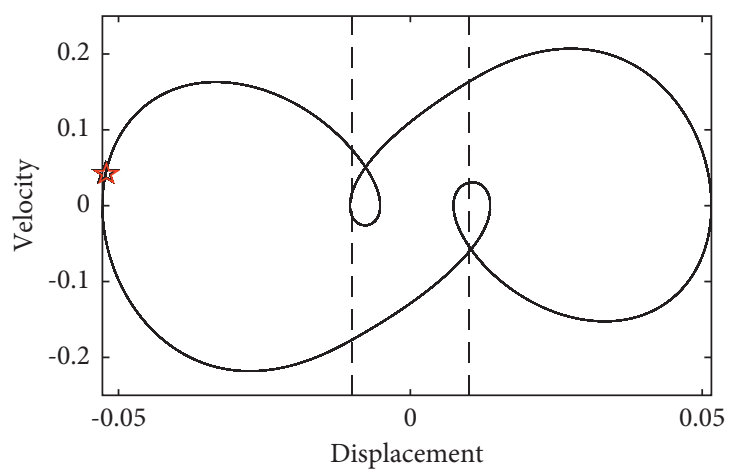

(b)

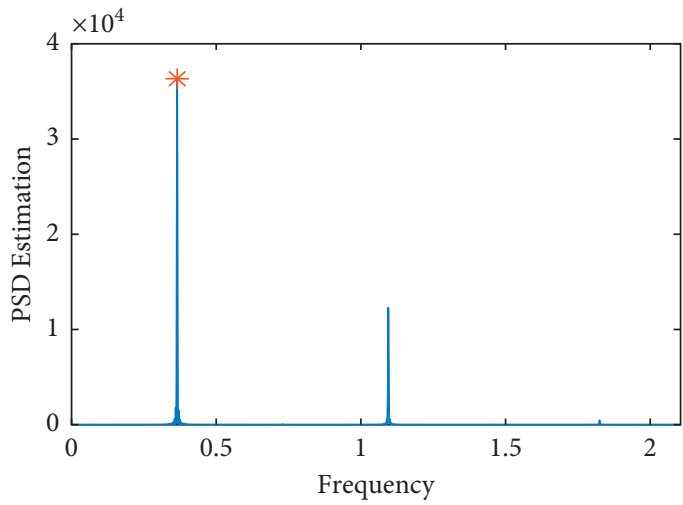

(d)

Figure 10: $f=0.365 \mathrm{~Hz}$, asymmetric period 1-1-2. (a) Time history graph. (b) Phase diagram. (c) Poincaré section. (d) Power spectral density diagram. 


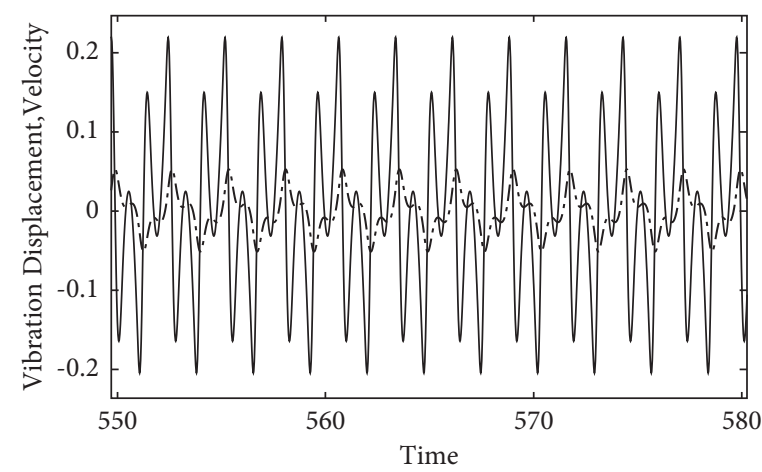

(a)

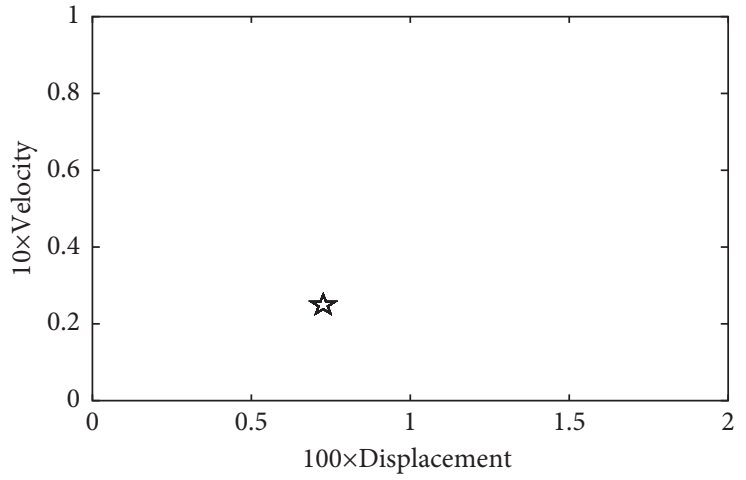

(c)

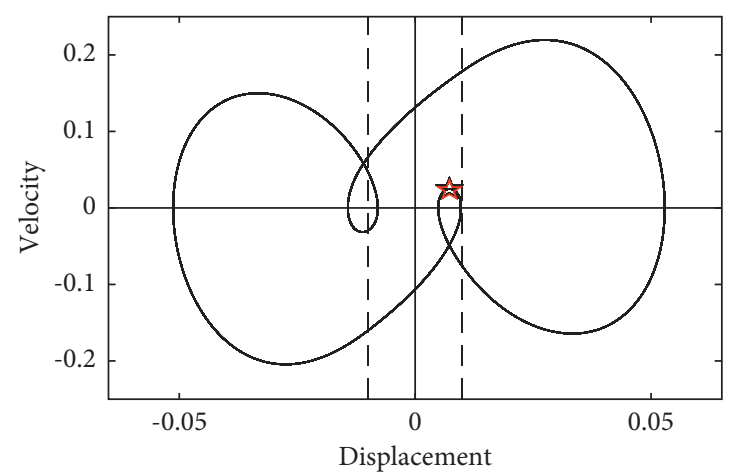

(b)

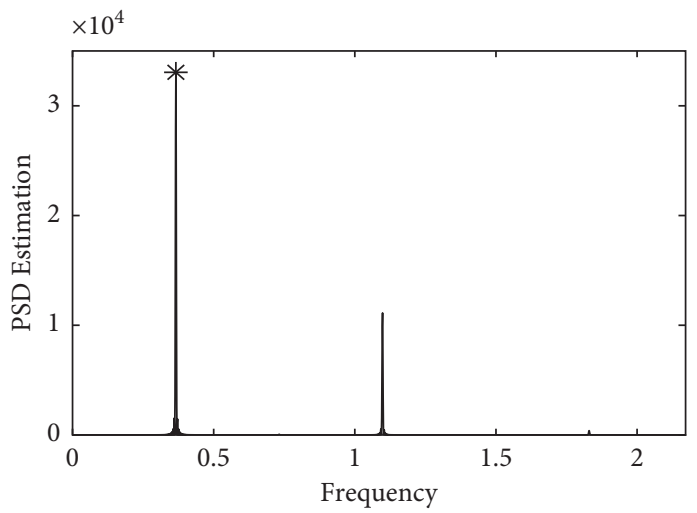

(d)

Figure 11: $f=0.366 \mathrm{~Hz}$, asymmetric period 1-2-1. (a) Time history graph. (b) Phase diagram. (c) Poincaré section. (d) Power spectral density diagram.

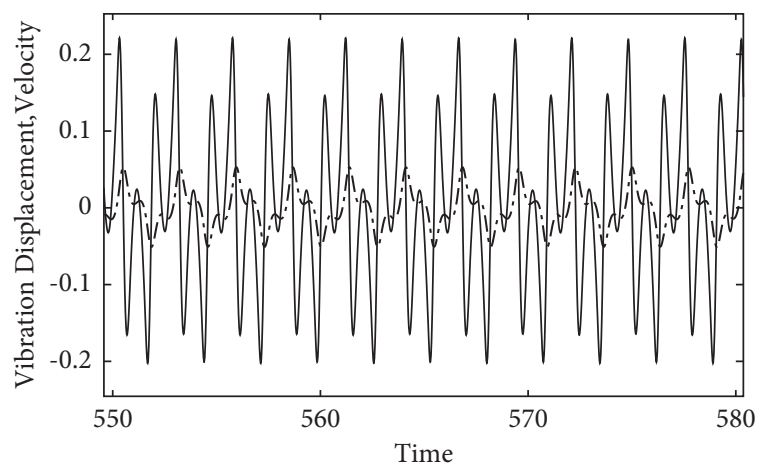

(a)

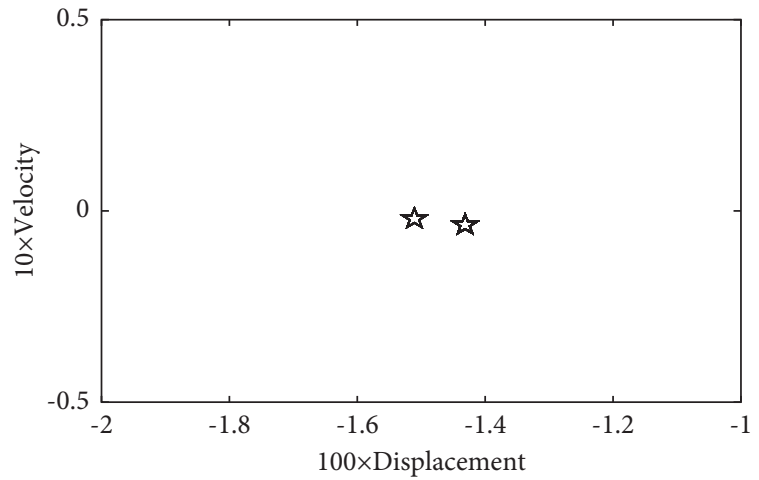

(c)

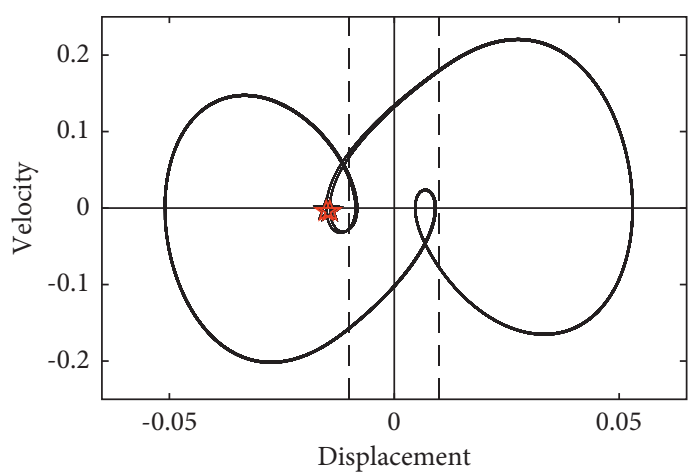

(b)

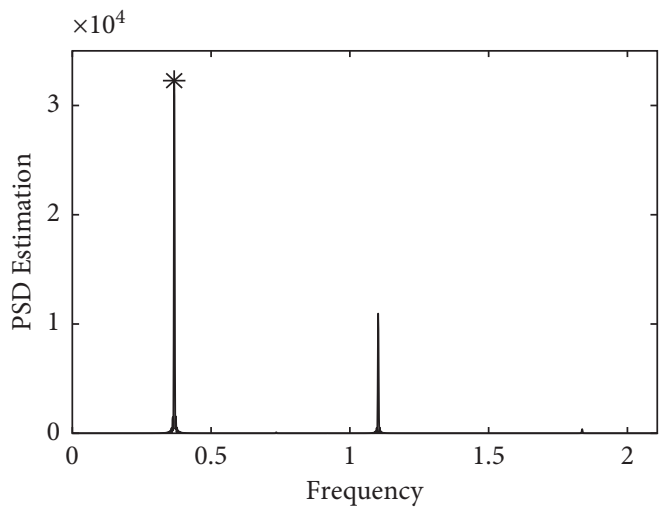

(d)

Figure 12: $f=0.3674 \mathrm{~Hz}$, asymmetric period 2-4-2. (a) Time history graph. (b) Phase diagram. (c) Poincaré section. (d) Power spectral density diagram. 


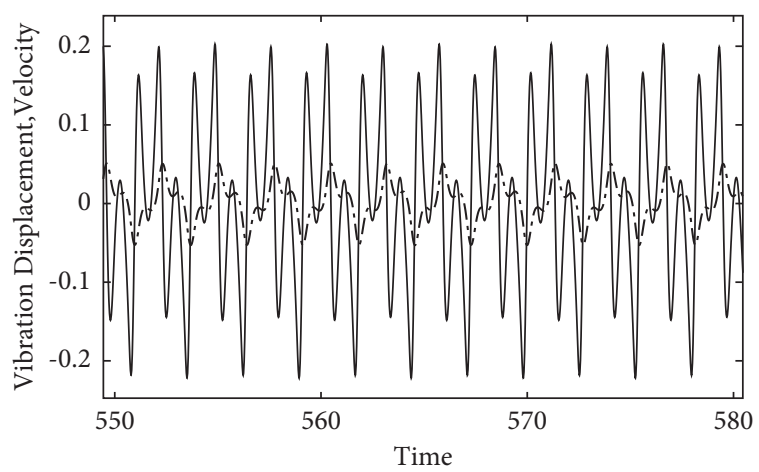

(a)

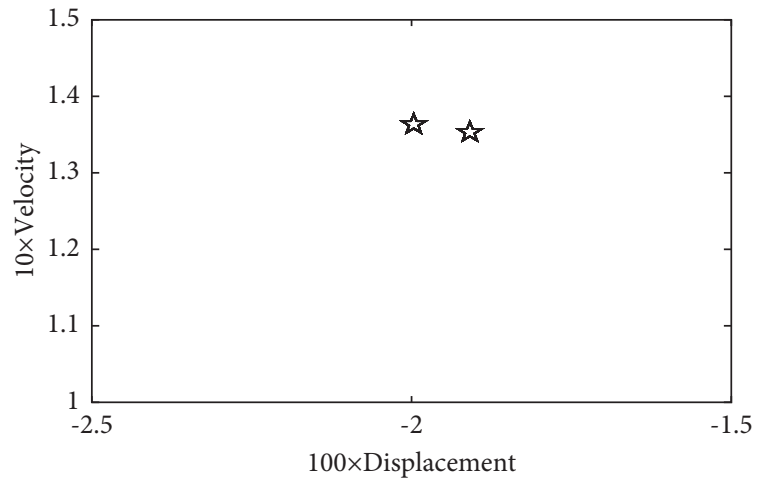

(c)

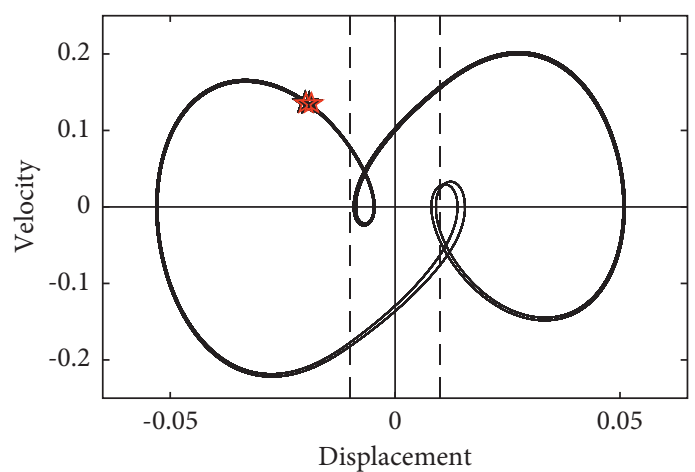

(b)

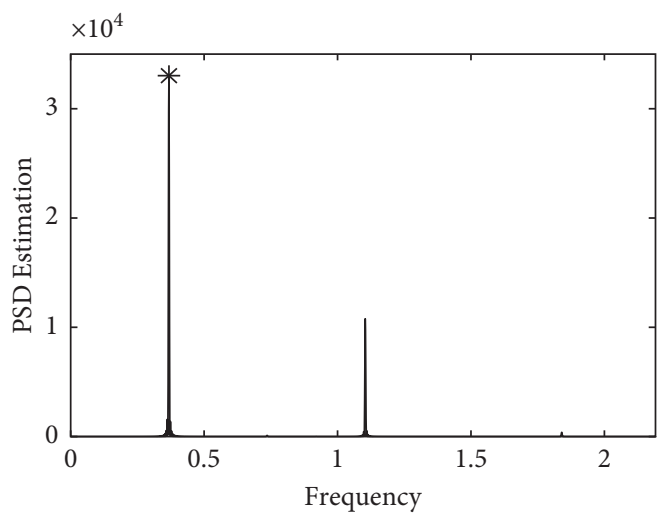

(d)

Figure 13: $f=0.368 \mathrm{~Hz}$, Asymmetric period 2-2-4. (a) Time history graph. (b) Phase diagram. (c) Poincaré section. (d) Power spectral density diagram.

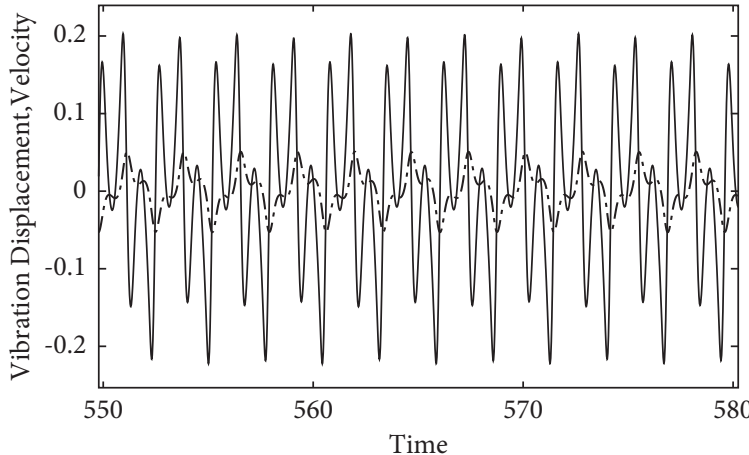

(a)

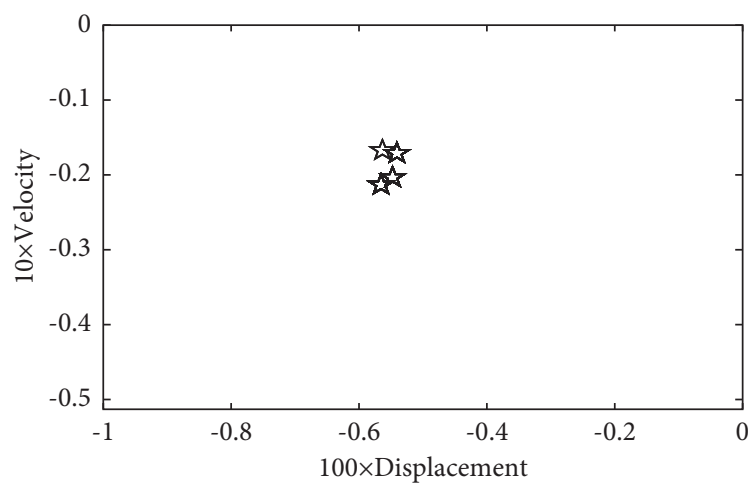

(c)

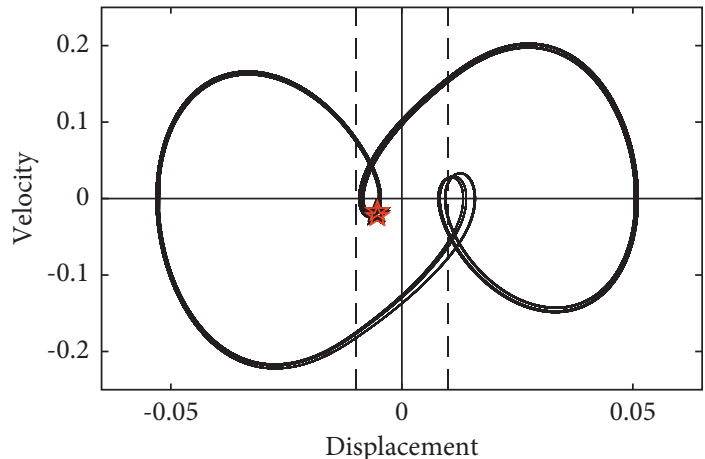

(b)

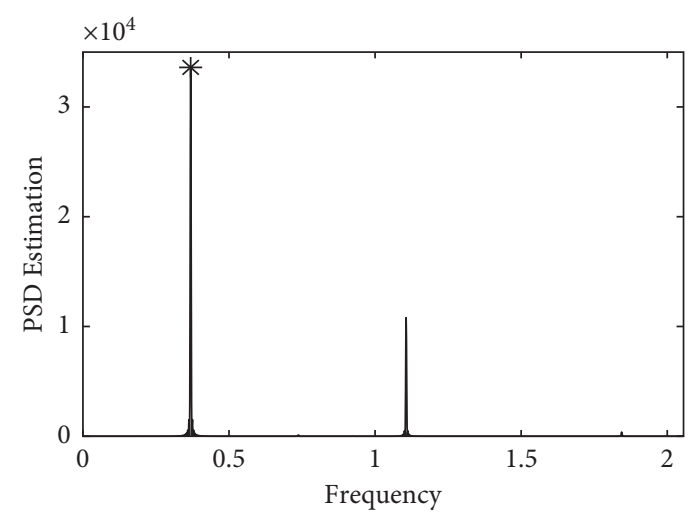

(d)

Figure 14: $f=0.3688 \mathrm{~Hz}$, asymmetric period 4-4-8. (a) Time history graph. (b) Phase diagram. (c) Poincaré section. (d) Power spectral density diagram. 


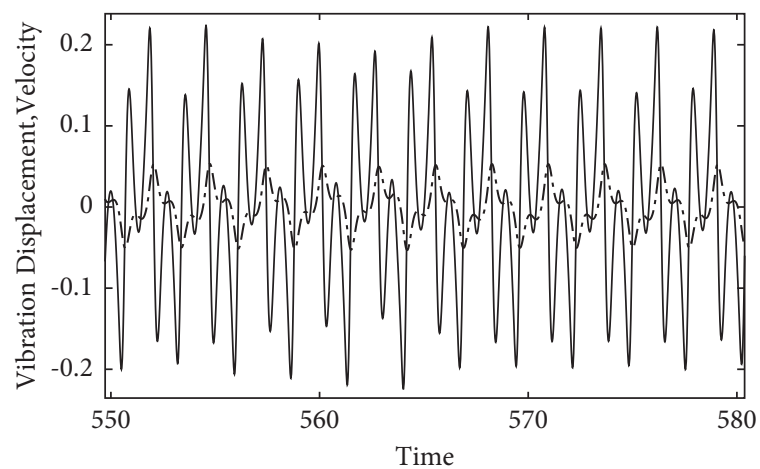

(a)

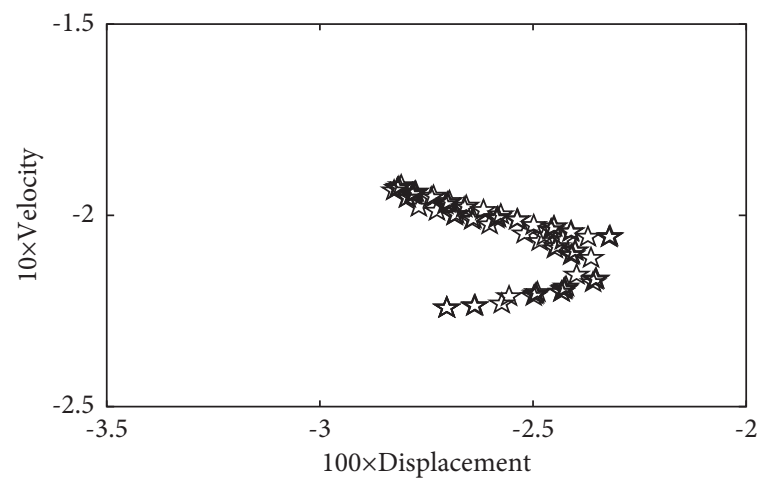

(c)

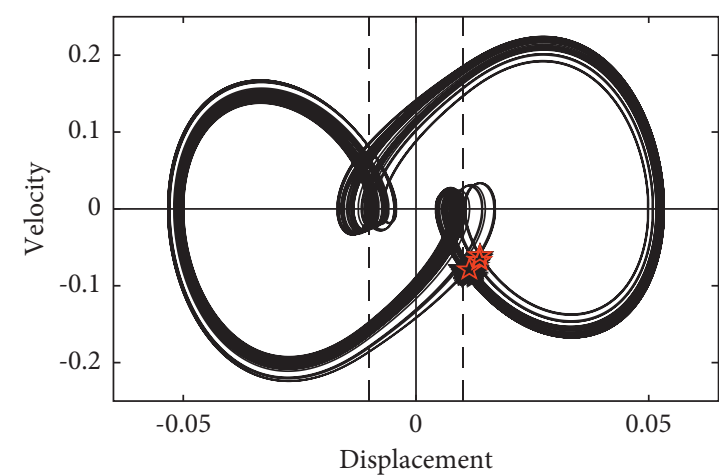

(b)

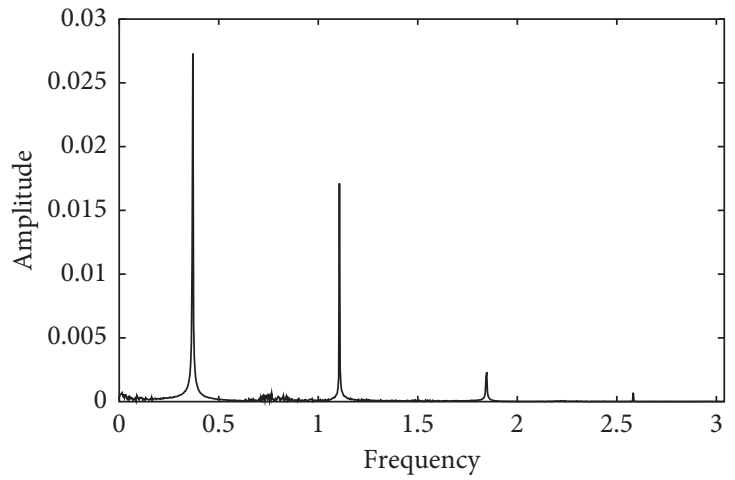

(d)

FIgUre 15: $f=0.370 \mathrm{~Hz}$, chaotic motion. (a) Time history graph. (b) Phase diagram. (c) Poincaré section. (d) Power spectral density diagram.

and the effects of clearance $b$, impact relative stiffness $k_{0}$, and damping ratio $\zeta$ on the dynamic response of the system are analyzed, respectively.

4.1. Influence of Hinge Clearance. Figure 16 shows the bifurcation diagram of the system varies with the hinge clearance at the excitation frequency $f=0.402,0.414$, $0.426 \mathrm{~Hz}$. Compared with the response of the system at different frequencies, the initial collision velocity of the system is positively correlated with the hinge clearance, and with the clearance value, the collision velocity decreases, and the impact vibration of the hinge is suppressed; the hinge clearance also makes the system produce mirror jumping and bifurcation, from period doubling to chaos, but at both ends of the clearance range, the system presents stable single period motion, and the system is unstable [39]. With the increase of the excitation frequency, the motion in the bifurcation diagram tends to be simple, and the chaotic window decreases gradually. When the excitation frequency increases, the only periodic motion is left in the bifurcation diagram. For the vibration response of articulated structures, it is necessary to design the structural parameters reasonably to avoid the low-frequency complex motion and realize the structural vibration suppression control.

4.2. Influence of Contact Stiffness of Hinge. Figure 17 shows the bifurcation diagram of the system varying with the contact stiffness of the hinge at the excitation frequency. By comparing the response of the system at different frequencies, it is found that the system with the change of contact stiffness has complex dynamic behaviors such as period-doubling bifurcation, edge trimming bifurcation, and mirror jumping transformation, and the evolution law of the dynamic response of the system with the change of stiffness is similar: at the left end of the bifurcation diagram, with the increase of stiffness, the system moves from single period motion to mirror jumping transformation and bifurcation; at the right end of the bifurcation diagram, with the decrease of the stiffness, the system directly changes from single period motion to chaos. The effect of frequency on the bifurcation diagram is not significant.

4.3. Influence of Damping Ratio on Beam Structure. Figure 18 shows the bifurcation diagram of the system varying with the damping ratio of the beam structure at the excitation frequency. By comparing the bifurcation diagrams under different frequencies with the decrease of damping ratio, the system experienced a jump mutation from single period motion to chaos through period-doubling bifurcation. The excitation frequency makes the bifurcation diagram shift as a whole; the period-doubling window at the small damping end is compressed when the frequency increases. The results show that the large damping of the structure accelerates the energy dissipation of the impact vibration and makes the motion response of the structure tend to be stable. 


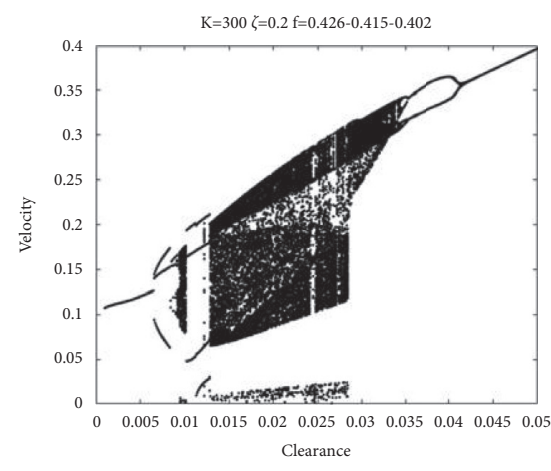

(a)

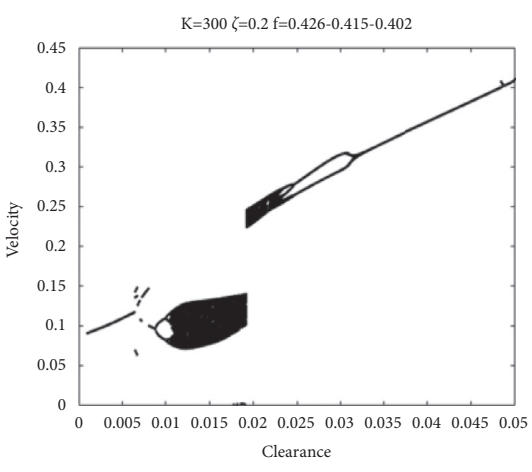

(b)

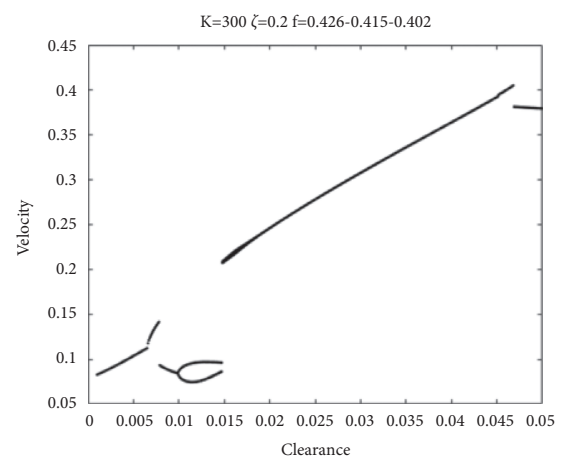

(c)

Figure 16: The effect of hinge clearance. (a) $f=0.402 \mathrm{~Hz}$, (b) $f=0.415 \mathrm{~Hz}$, and (c) $f=0.426 \mathrm{~Hz}$.

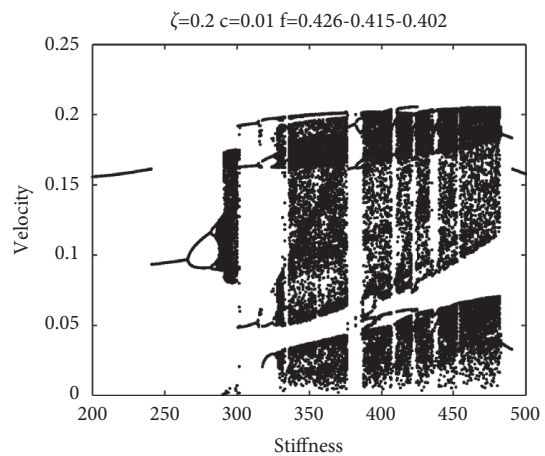

(a)

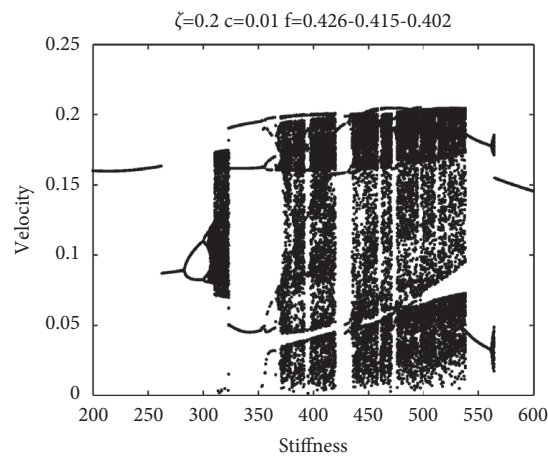

(b)

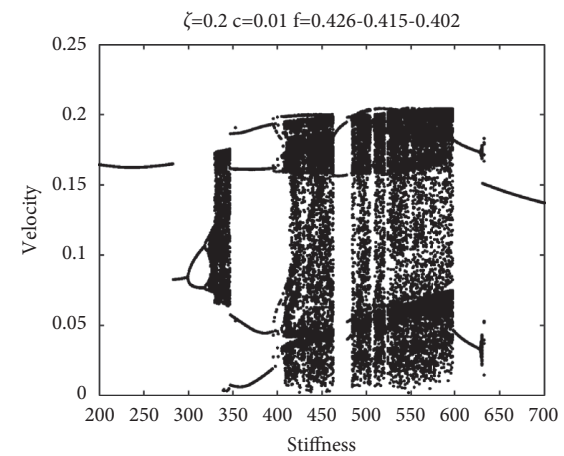

(c)

Figure 17: Influence of hinge contact stiffness. (a) $f=0.402 \mathrm{~Hz}$, (b) $f=0.415 \mathrm{~Hz}$, and (c) $f=0.426 \mathrm{~Hz}$.

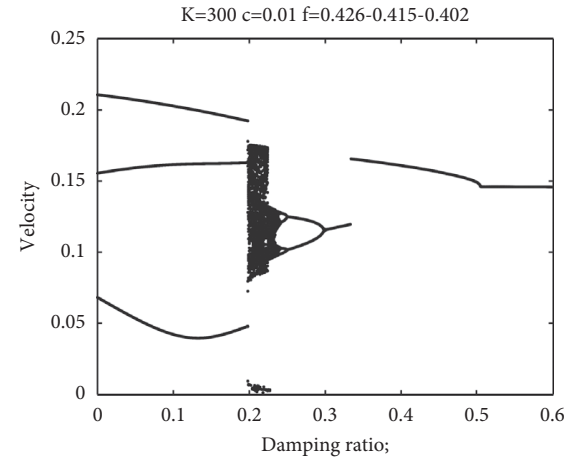

(a)

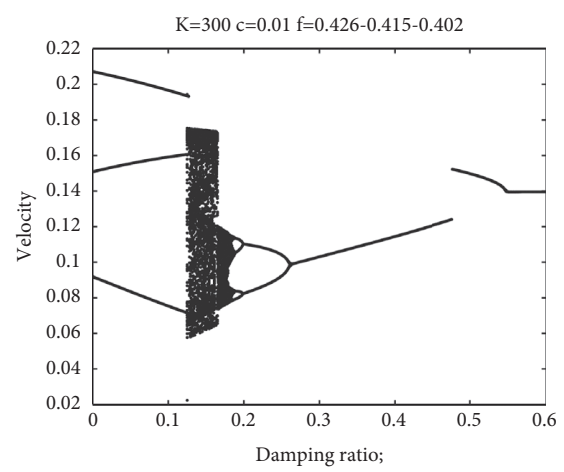

(b)

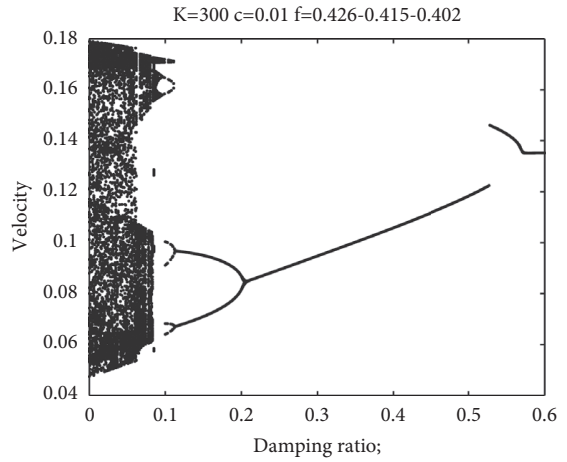

(c)

Figure 18: Influence of beam structure damping ratio. (a) $f=0.402 \mathrm{~Hz}$, (b) $f=0.415 \mathrm{~Hz}$, and (c) $f=0.426 \mathrm{~Hz}$.

\section{Conclusion}

Based on the equivalent dynamic model of the clearance hinge, the differential formula of the system motion is established, and the numerical simulation is carried out by MATLAB programming. The bifurcation and chaos phenomena with the excitation frequency under the harmonic excitation of the clearance hinge are simulated and analyzed, and the effects of the hinge clearance, the contact stiffness, and the damping ratio of the beam structure on the system motion are also studied. The following conclusions are drawn: 
(1) The dynamic response of the hinge system is complex with the change of excitation frequency. In the bifurcation diagram, the periodic motion has a sudden change of impact state and a mirror image jump transformation. The path from periodic motion to chaos is mainly divided into two types: period-doubling bifurcation and edging bifurcation.

(2) The way of system bifurcation to chaos is usually as follows: the edge scrubbing first destroys the symmetry of the motion of the system and then bifurcates into chaos along with the heap transformation, but there can also be wiping edges directly into chaos.

(3) For the established single-degree-of-freedom collision vibration system, within a certain range of parameters, the initial collision velocity of the system is positively correlated with the hinge clearance; the larger the clearance is, the more intense the impact vibration is; the contact stiffness of the hinge mainly affects the number of collisions in the periodic motion; generally, under the same excitation frequency, the greater the stiffness, the more the number of collisions; the damping ratio of the beam structure is related to the energy dissipation and transfer of the system, and the large damping ratio usually limits the complex motion of the system.

\section{Data Availability}

The data of characteristics and parameters of the bilateral restraint vibration-impact system used to support the findings of this study are included within the article.

\section{Conflicts of Interest}

The authors declare that they have no conflicts of interest.

\section{Acknowledgments}

This work was financially supported by China Agriculture Research System (CARS-04-ps-25), Ph.D. Research Startup Foundation of Henan Agricultural University (30500976), Postdoctoral Research on Agricultural Engineering Mobile Station of Henan Agricultural University (10400103), and the Science and Technology Project of Henan Province (212102110219).

\section{References}

[1] G. Shi and S. N. Atluri, "Nonlinear dynamic response of frame-type structures with hysteretic damping at the joints," AIAA Journal, vol. 30, no. 1, pp. 234-240, 1992.

[2] F. C. Moon and G. X. Li, "Experimental study of chaotic vibrations in a pin-jointed space truss structure," AIAA Journal, vol. 28, no. 5, pp. 915-921, 1990.

[3] H. S. Tzou, "Non-linear joint dynamics and controls of jointed flexible structures with active and viscoelastic joint actuators," Journal of Sound and Vibration, vol. 143, no. 3, pp. 407-422, 1990.
[4] W. Wang, "Advances and trends of non-linear dynamics of space joint-dominated structure," Advances in Mechanics, vol. 36, no. 2, pp. 233-238, 2006.

[5] J. Zhang, "Influence analysis of joints on nonlinear dynamic characteristics of articulated structures," Acta Aeronautica et Astronautica Sinica, vol. 35, no. 5, pp. 1433-1445, 2014.

[6] J. Knudsen and A. R. Massih, "Dynamic stability of weakly damped oscillators with elastic impacts and wear," Journal of Sound and Vibration, vol. 263, no. 1, pp. 175-204, 2003.

[7] R. I. Leine, "Non-smooth stability analysis of the parametrically excited impact oscillator," International Journal of Non-linear Mechanics, vol. 47, no. 9, pp. 1020-1032, 2012.

[8] N. Humphries and P. T. Piiroinen, "A discontinuity-geometry view of the relationship between saddle-node and grazing bifurcations," Physica D: Nonlinear Phenomena, vol. 241, no. 22, pp. 1911-1918, 2012.

[9] G. Stefani, M. De Angelis, and U. Andreaus, "Numerical study on the response scenarios in a vibro-impact single-degree-offreedom oscillator with two unilateral dissipative and deformable constraints," Communications in Nonlinear Science and Numerical Simulation, vol. 99, Article ID 105818, 2021.

[10] H. Gritli and S. Belghith, "Diversity in the nonlinear dynamic behavior of a one-degree-of-freedom impact mechanical oscillator under OGY-based state-feedback control law: order, chaos and exhibition of the border-collision bifurcation," Mechanism and Machine Theory, vol. 124, pp. 1-41, 2018.

[11] G. W. Luo, X. F. Zhu, and Y. Q. Shi, "Dynamics of a twodegree-of freedom periodically-forced system with a rigid stop: d," Journal of Sound and Vibration, vol. 334, pp. 338362, 2015.

[12] H. Jiang, A. S. E. Chong, Y. Ueda, and M. Wiercigroch, "Grazing-induced bifurcations in impact oscillators with elastic and rigid constraints," International Journal of Mechanical Sciences, vol. 127, pp. 204-214, 2017.

[13] J. Chen, F. Min, Q. Jin, and B. Ye, "Coexistence, bifurcation and chaos of a periodically forced duffing system with absolute nonlinearity," The European Physical Journal - Special Topics, vol. 228, no. 6, pp. 1405-1419, 2019.

[14] X. Lyu, Q. Gao, and G. Luo, "Dynamic characteristics of a mechanical impact oscillator with a clearance," International Journal of Mechanical Sciences, vol. 178, Article ID 105605, 2020.

[15] W. Zhang, F. Min, J. Chen, and Y. Dou, "Discontinuous dynamic analysis of a modified duffing-Rayleigh system with a pqf,” IEEE ACCESS, vol. 8, pp. 32312-32320, 2020.

[16] U. Andreaus and M. De, "Nonlinear dynamic response of a base-excited SDOF oscillator with double-side unilateral constraints," Nonlinear Dynamics, vol. 84, no. 3, pp. 1447-1467, 2016.

[17] U. Andreaus, P. Baragatti, M. De Angelis, and S. Perno, "A preliminary experimental study about two-sided impacting SDOF oscillator under harmonic excitation," Journal of Computational and Nonlinear Dynamics, vol. 12, no. 6, 2017.

[18] G. Stefani, M. De Angelis, and U. Andreaus, "Scenarios in the experimental response of a vibro-impact single-degree-offreedom system and numerical simulations," Nonlinear Dynamics, vol. 103, no. 4, pp. 3465-3488, 2021.

[19] X. H. Lyu, Q. F. Gao, and G. W. Luo, "Dynamic characteristics of a mechanical impact oscillator with a clearance," International Journal of Mechanical Sciences, vol. 178, 2020.

[20] G. F. Li, S. P. Wu, H. B. Wang, and W. C. Ding, "Global dynamics of a non-smooth system with elastic and rigid impacts and dry friction," Communications in Nonlinear Science and Numerical Simulation, vol. 95, 2021. 
[21] G. F. Li, S. P. Wu, H. B. Wang, J. Sun, and W. C. Ding, "Global behavior of a simplified model for the micro-vibration molding machine in parameter-state space," Mechanism and Machine Theory, vol. 154, 2021.

[22] J. J. Fan, J. Cao, S. L. Chen, C. J. Dou, and S. Xue, "Discontinuous dynamic analysis of a class of three degrees of freedom mechanical oscillatory systems with dry friction and one-sided rigid impact," Mechanism and Machine Theory, vol. 151, 2020.

[23] C. L. Li, J. J. Fan, Z. X. Yang, and S. Xue, "On discontinuous dynamical behaviors of a 2-DOF impact oscillator with friction and a periodically forced excitation," Mechanism And Machine Theory, vol. 135, pp. 81-108, 2019.

[24] J. J. Fan, T. Y. Liu, and S. L. Chen, "Analysis of dynamical behaviors of a 2-DOF friction-induced oscillator with onesided impact on a conveyor belt," Nonlinear Dynamics, vol. 97, no. 1, pp. 797-830, 2019.

[25] G. L. Wen, S. Yin, H. D. Xu, S. J. Zhang, and Z. Y. Lv, "Analysis of grazing bifurcation from periodic motion to quasi-periodic motion in impact-damper systems," Chaos Solitons \& Fractals, vol. 83, pp. 112-118, 2016.

[26] S. Yin, J. C. Ji, and G. L. Wen, "Complex near-grazing dynamics in impact oscillators," International Journal of Mechanical Sciences, vol. 156, pp. 106-122, 2019.

[27] J. F. Shi, X. F. Gou, and L. Y. Zhu, "Modeling and analysis of a spur gear pair considering multi-state mesh with time-varying parameters and backlash," Mechanism and Machine Theory, vol. 134, pp. 582-603, 2019.

[28] G. W. Luo, L. Ma, and X. H. Lv, "Dynamic analysis and suppressing chaotic impacts of a two-degree-of-freedom oscillator with a clearance," Nonlinear Analysis: Real World Applications, vol. 10, no. 2, pp. 756-778, 2009.

[29] F. Turki, H. Gritli, and S. Belghith, "An LMI-based design of a robust state-feedback control for the master-slave tracking of an impact mechanical oscillator with double-side rigid constraints and subject to bounded-parametric uncertainty," Communications in Nonlinear Science and Numerical Simulation, vol. 82, 2020.

[30] S. Yin, G. L. Wen, and X. Wu, "Suppression of grazing-induced instability in single degree-of-freedom impact oscillators," Applied Mathematics And Mechanics-English Edition, vol. 40, no. 1, pp. 97-110, 2018.

[31] H. Gritli, "Robust master-slave synchronization of chaos in a one-sided 1-DoF impact mechanical oscillator subject to parametric uncertainties and disturbances," Mechanism and Machine Theory, vol. 142, 2019.

[32] H. D. Xu, S. Yin, G. L. Wen, S. J. Zhang, and Z. Y. Lv, "Discrete-in-time feedback control of near-grazing dynamics in the two-degree-of-freedom vibro-impact system with a clearance," Nonlinear Dynamics, vol. 87, no. 2, pp. 1127-1137, 2017.

[33] S. Yin, J. C. Ji, G. L. Wen, and X. Wu, "Use of degeneration to stabilize near grazing periodic motion in impact oscillators," Communications in Nonlinear Science and Numerical Simulation, vol. 66, pp. 20-30, 2019.

[34] H. Gritli and S. Belghith, "Walking dynamics of the passive compass-gait model under OGY-based state-feedback control: Rise of the Neimark-Sacker bifurcation," Chaos Solitons \& Fractals, vol. 110, pp. 158-168, 2018.

[35] H. Gritli and S. Belghith, "Walking dynamics of the passive compass-gait model under OGY-based control: emergence of bifurcations and chaos," Communications in Nonlinear Science and Numerical Simulation, vol. 47, pp. 308-327, 2018.
[36] H. Gritli and S. Belghith, "Walking dynamics of the passive compass-gait model under OGY-based state-feedback control: analysis of local bifurcations via the hybrid Poincare map," Chaos Solitons \& Fractals, vol. 98, pp. 72-87, 2017.

[37] Y. K. Shen, S. Yin, G. L. Wen, and H. D. Xu, "Feedback control of grazing-induced chaos in the single-degree-of-freedom impact oscillator," Journal of Computational and Nonlinear Dynamics, vol. 131 page, 2017.

[38] S. Kumar, Narayanan, and S. Gupta, "Bifurcation analysis of a stochastically excited vibro-impact Duffing-Van der Pol oscillator with bilateral rigid barriers," International Journal of Mechanical Sciences, vol. 127, pp. 103-117, 2017.

[39] X. Lyu, X. Zhu, Q. Gao, and G. Luo, “Two-parameter bifurcations of an impact system under different damping conditions," Chaos, Solitons \& Fractals, vol. 138, Article ID 109972, 2020. 